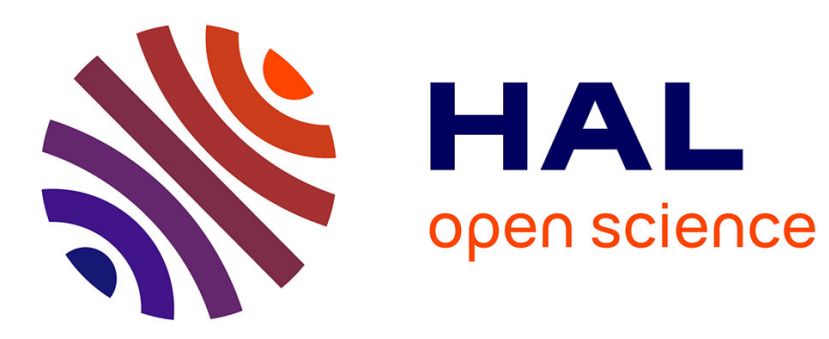

\title{
Bandpass NGD Time-Domain Experimental Test of Double-li Microstrip Circuit
}

\author{
Remy Vauche, Rym Assila Belhadj Mefteh, Fayrouz Haddad, Wenceslas \\ Rahajandraibe, Fayu Wan, Sebastien Lallechere, Glauco Fontgalland, Preeti \\ Thakur, Atul Thakur, Blaise Ravelo
}

\section{To cite this version:}

Remy Vauche, Rym Assila Belhadj Mefteh, Fayrouz Haddad, Wenceslas Rahajandraibe, Fayu Wan, et al.. Bandpass NGD Time-Domain Experimental Test of Double-li Microstrip Circuit. IEEE Design \& Test, 2022, pp.1-1. 10.1109/MDAT.2021.3103457 . hal-03494716

\section{HAL Id: hal-03494716 https://hal.science/hal-03494716}

Submitted on 20 Dec 2021

HAL is a multi-disciplinary open access archive for the deposit and dissemination of scientific research documents, whether they are published or not. The documents may come from teaching and research institutions in France or abroad, or from public or private research centers.
L'archive ouverte pluridisciplinaire HAL, est destinée au dépôt et à la diffusion de documents scientifiques de niveau recherche, publiés ou non, émanant des établissements d'enseignement et de recherche français ou étrangers, des laboratoires publics ou privés. 


\title{
Bandpass NGD Time-Domain Experimental Test of Double-li Microstrip Circuit
}

\author{
Rémy Vauché, Member, IEEE, Rym Assila Belhadj Mefteh, Fayrouz Haddad, Member, IEEE, \\ Wenceslas Rahajandraibe, Member, IEEE, Fayu Wan, Member, IEEE, Sébastien Lalléchère, Member, \\ IEEE, Glauco Fontgalland, Senior Member, IEEE, Preeti Thakur, Atul Thakur and Blaise Ravelo, \\ Member, IEEE
}

\begin{abstract}
This paper details a time-domain (TD) test to visualize impacts on the double-li microstrip circuit behavior of its bandpass (BP) negative group delay (NGD). To determine the central frequency and the bandwidth of the input signal to use during the TD test, a frequency domain (FD) S-parameter analysis of the circuit has been done. This preliminary analysis, carried out first with the aid of simulations and then with the aid of measurements, shows a NGD for a frequency band of $15 \mathrm{MHz}$ (resp. $8 \mathrm{MHz}$ ) around $2.345 \mathrm{GHz}$ (resp. $2.364 \mathrm{GHz}$ ). To observe in the TD the NGD impact around $2.345 \mathrm{GHz}$, TD experimentations have been performed using a $2.345 \mathrm{GHz}$ sine carrier shaped by Gaussian pulses. During these TD tests, the BP NGD signature was verified thanks to the output envelopes which presents rising and falling edges in time-advance compared to the input ones. It was also experimentally shown that the output is normally delayed when the input sine carrier is outside of the li-circuit NGD frequency bands.
\end{abstract}

Index Terms-Bandpass (BP) negative group delay (NGD), Microwave circuit, Time-domain, Test method, li-topology.

\section{INTRODUCTION}

$\mathrm{T}$ HE GROUP DELAY (GD) estimation [1], which can be defined as the signal envelope delay estimation, is one of the challenging tasks to perform an efficient time-domain (TD) analysis of microwave structures based on transmission lines (TLs) [2-3]. Indeed, whereas the GD is usually measured in the frequency domain (FD) using a vector network analyzer (VNA)

This work was supported in part by the Jiangsu Innovation and Enterprise Group Talents Plan 2015 under Grant SRCB201526, the National Natural Science Foundation of China under Grants 61601233 and 61750110535, the NSF of Jiangsu under Grants BK20150918, and the Priority Academic Program Development of Jiangsu Higher Education Institutions.

R. Vauche, R. Assila Belhadj Mefteh, F. Haddad, and W. Rahajandraibe are with the Provence Institute of Materials Microelectronics and Nanosciences (IM2NP), Aix-Marseille University, Batiment Fermi, 5 rue Enrico Fermi, 13453 Marseille Cedex 13, France (e-mail: remy.vauche@univ-amu.fr, rym.assila@im2np.fr, fayrouz.haddad@im2np.fr, wenceslas.rahajandraibe @univ-amu.fr).

F. Wan, and B. Ravelo are with the Electronics and Information Engineering College, Nanjing University of Information Science and Technology, N219, Ningliu Rd, Pukou, Nanjing China, 210044 (e-mail: fayu.wan@nuist.edu.cn, blaise.ravelo@nuist.edu.cn).

S. Lalléchère is with the Institut Pascal and Universite Clermont Auvergne, 5A rue d'Allagnat, 63000, Clermont-Ferrand, France (e-mail: sebastien.lallechere@uca.fr).

G. Fontgalland is with the Federal University of Campina Grande, Rua Aprigio Veloso, 890, Campina Grande/PB, 58429-900, Brazil, (e-mail: fontgalland@dee.ufcg.edu.br)

P. Thakur and A. Thakur are with the Amity University Haryana, Gurgaon, 122413, India (e-mail: pthakur@ggn.amity.edu, athakur1@ggn.amity.edu). from the transmission parameter, the GD estimation in the TD from the envelope measurement [4] of modulated signals [2] requires investigations. This is all the more important that a TD analysis is particularly interesting to understand the impact of the NGD which can be obtained with several coupled line (CL) structures [5-6].

Recent studies report that unfamiliar negative group delay (NGD) functions have been designed with CL-based microstrip circuits [7-8]. It has been emphasized from a FD analysis that the CL li-topology [5] constituted by two parallel I-shape lines is susceptible to have a NGD in function of the CL effective length. However, the interpretation of the NGD function remains an open question for non-specialist electronic and microwave design engineers.

One of the easiest manners to describe an electronic function in terms of NGD is based on the analogy with the filters [9]. Using it, a low-pass NGD function has a NGD for low frequencies and a positive GD (PGD) for high frequencies. The behavior of a low-pass NGD function has been recently illustrated with TD measurement [10]. It was also emphasized that rising and falling edges of the output signal have been observed in time-advance compared to the input ones when baseband signals are considered [11]. But so far, bandpass (BP) NGD functions which can be obtained with the li-topology and the associate TD behavior require further research works.

For this reason, the methodology of BP NGD TD measurement is investigated in the present study and is applied to a microstrip circuit based on the li-topology discussed in [7]. It can be noted that the designed circuit has the particularity to have two frequency bands for which the GD is negative. Thus, the paper is organized as follows. Section II presents in a first time the specifications of the unfamiliar BP NGD function, and in a second time the design and performances of the circuit under test (CUT) based on FD simulations and measurement. Next, section III focuses on the developed methodology for the TD measurement of BP NGD functions. Then, results from the TD test illustrating the BP NGD signature of the CUT are discussed. Finally, section IV concludes the paper.

\section{BP NGD PROTOTYPING AND FD ANALYSIS}

This section begins with the introduction of the unfamiliar BP NGD ideal function. Next, the design process of the double-li CUT is described. Then, the BP NGD aspect is validated using a FD analysis based on S-parameter measurements. 


\section{A. BP NGD Ideal Specifications}

A circuit can be generalized by a two-port black box as shown in Fig. 1(a). Using the Laplace variable $s=j \omega=j 2 \pi f$, its S-matrix can be written as follows:

$$
[S(s)]=\left[\begin{array}{ll}
S_{11}(s) & S_{12}(s) \\
S_{21}(s) & S_{22}(s)
\end{array}\right] .
$$

The GD associated to the circuit is also defined by:

$$
G D(\omega)=-\partial\left\{\arg \left[S_{21}(j \omega)\right]\right\} / \partial \omega .
$$

The circuit can be considered as a BP NGD function if it presents a GD $<0$ for a bandwidth $B_{n}$ around a frequency $f_{n}$ as illustrated in Fig. 1(b), $f_{n}$ being the frequency for which a local GD minimum denoted $G D_{n}$ is obtained. The frequencies around $f_{n}$ for which the GD is equal to 0 are also called cut-off frequencies by analogy to filters.

To perform the TD BP NGD analysis, an input signal $V_{\text {in }}$ presenting a spectrum centered on $f_{n}$ with a bandwidth $B \leq B_{n}$ is required. This bandwidth $B$ can be defined as a function of an attenuation $a$ with respect to the maximal spectrum magnitude $V_{\max }$ as shown in Fig. 1(b). Thus, if it is assumed that the circuit is excited by an input signal represented by its Fourier transform $V_{i n}(j \omega)$, the attenuation of the input signal spectrum at the NGD cut-off angular frequencies $\omega_{C k}$ (with $k=1 . . \mathrm{K} ; \mathrm{K}$ being equal to 2 for a single passband NGD function) defined as:

$$
a=\left|V_{i n}\left(j \omega_{C k}\right)\right| / \max \left[V_{i n}(j \omega)\right]
$$

must also be chosen low enough to realize the TD analysis (for example $\mathrm{a}=0.1$ ).

\section{B. Design and Implementation of the BP NGD Prototype}

The NGD prototype has been designed with two cascaded stages of the li-microstrip topology proposed in [7]. The TLs physical widths are the same and chosen equal to $1.09 \mathrm{~mm}$. The li-lines physical lengths and interspaces have been optimized using HFSS ${ }^{\circledR}$ simulations to operate as a dual-band NGD function with arbitrary chosen NGD center frequencies between $f_{\min }=2.3 \mathrm{GHz}$ and $f_{\max }=2.4 \mathrm{GHz}$, and with insertion losses at the NGD center frequencies better than $5 \mathrm{~dB}$. By denoting the effective permittivity $\varepsilon_{\text {reff, }}$, the physical length of the li-shape microstrip line $d_{n}$ can be initially calculated as a function of the NGD targeted center frequency $f_{n}$ using:

$$
d_{n}=c /\left(2 f_{n} \sqrt{\varepsilon_{\text {reff }}}\right) \text {. }
$$

The CUT 3-D design and the photograph of the prototype implemented on $\mathrm{Cu}$-metalized Rogers 3210 dielectric substrate are respectively shown in Fig. 2(a) and Fig. 2(b). It can be noted that the CL with physical length $d_{1}$ (resp. $d_{2}$ ) has a coupling coefficient $-29.5 \mathrm{~dB}$ (resp. $-31.5 \mathrm{~dB}$ ).

The BP NGD aspect has initially been validated using frequency domain S-parameter simulations, and experimentally verified with the help of a Vector Network Analyzer (VNA) thanks to (2). Thus, the double-li NGD CUT, previously shown in Fig. 2(b), has been measured with the Rohde \& Schwarz ZNB 20 VNA from $100 \mathrm{kHz}$ to $20 \mathrm{GHz}$ after a Short-Open-Load-Through (SOLT) calibration. The experimental setup is shown in Fig. 2(c).

\section{NGD Design Validation in the FD}

The following validation study is based on the comparison between the FD simulated ("Sim.") and measured ("Meas.") results which have been carried out in the frequency band from $f_{\min }=2.3 \mathrm{GHz}$ to $f_{\max }=2.4 \mathrm{GHz}$. The GDs and S-parameters from simulation and measurement are plotted in Figs. 3. As depicted in Fig. 3(a), the CUT exhibits two NGD center frequencies $\left(f_{1}=2.345 \mathrm{GHz}\right.$ and $\left.f_{2}=2.364 \mathrm{GHz}\right)$ and measurement results confirms that the double-li CUT behaves as a dual-band BP NGD function. Nevertheless, FD response differences between simulations, and measurement can be observed and are mainly due to the substrate permittivity dispersion in addition to inaccuracies between its ideal value and its real one, but equally to the skin effect of the metallization conductor line, the fabrication tolerances, and the numerical computation inaccuracies. Figs. 3(b) and 3(c) illustrate that the insertion and reflection losses are better than $3.5 \mathrm{~dB}$ and $9 \mathrm{~dB}$ at the NGD center frequencies. Table I summarizes simulated, and measured results.

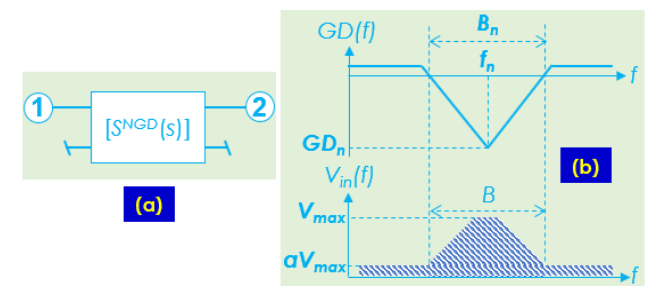

Fig. 1. (a) Two-port black box and (b) the typical BP NGD response with the input time domain test signal spectrum.

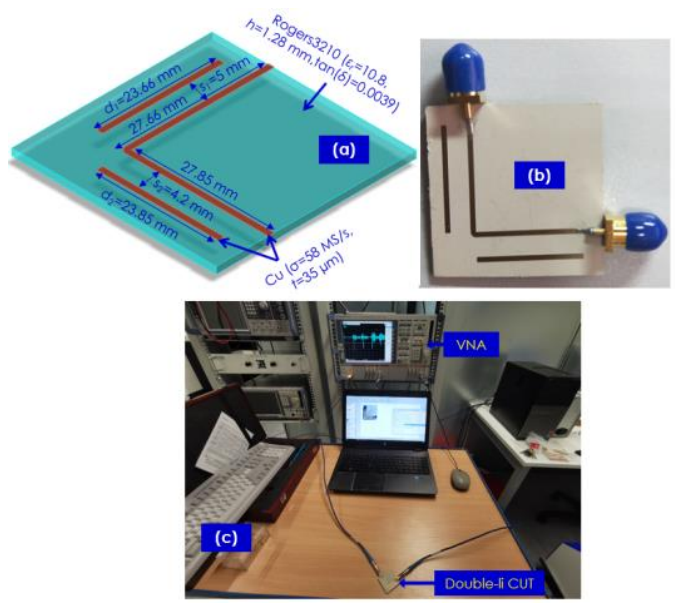

Fig. 2. Double li-CUT (a) 3-D design, (b) photograph, and (c) experimental setup.

TABLE I

COMPARISON BETWEEN FREQUENCY DOMAIN SIMULATIONS AND MEASUREMENT

\begin{tabular}{c|c|c|c|c|c}
\hline \hline $\begin{array}{c}\text { Validation } \\
\text { method }\end{array}$ & $\begin{array}{c}f_{n} \\
(\mathrm{GHz})\end{array}$ & $\begin{array}{c}G D_{n} \\
(\mathrm{~ns})\end{array}$ & $\begin{array}{c}B_{n} \\
(\mathrm{MHz})\end{array}$ & $\begin{array}{c}S_{21}\left(f_{n}\right) \\
(\mathrm{dB})\end{array}$ & $\begin{array}{c}S_{11}\left(f_{n}\right) \\
(\mathrm{dB})\end{array}$ \\
\hline Simu. & 2.3475 & -4.55 & 10.0 & -2.60 & -12.02 \\
\hline Meas. & 2.345 & -4.44 & 15.0 & -3.64 & -9.38 \\
\hline Simu. & 2.3638 & -2.73 & 8.75 & -2.01 & -13.19 \\
\hline Meas. & 2.364 & -1.88 & 8.00 & -2.39 & -14.90 \\
\hline Simu. & 2.5 & 0.5354 & N/A* & -0.2024 & -32.84 \\
\hline Meas. & 2.5 & 0.748 & N/A* & -0.443 & -14.19 \\
\hline \hline
\end{tabular}



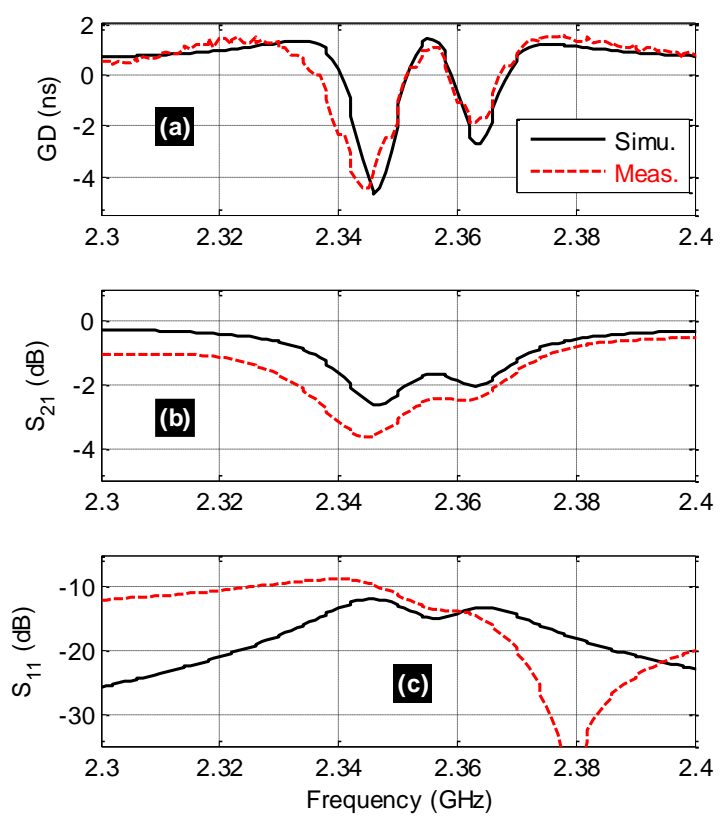

Fig. 3. Comparison of simulated and measured (a) GD, (b) $S_{21}$, and (c) $S_{11}$ of the double-li circuit prototype shown in Fig. 2(b).

Finally, from the present FD validation, the TD test of the double-li NGD circuit is investigated in the following section. For the oversight understanding of the TD NGD effect, the center frequency $f_{P G D}=2.5 \mathrm{GHz}$, for which a positive group delay (PGD) can be observed, is considered during the analysis.

\section{BP NGD TD EXPERIMENTAL TEST RESULTS OF THE DOUBLE li-MICROWAVE CIRCUIT}

After the CUT introduction, the methodology of BP NGD TD experimentation is described in the present section. Next, the method feasibility is verified with the double li prototype described in the previous section.

\section{A. Time Domain Test Methodology of the BP NGD Circuit}

The TD test of the BP NGD circuit can be formulated using the following steps.

- Step 1: The NGD CUT initial validation is based on the FD analysis in order to verify the specifications illustrated in Fig. 1(b). S-parameters measurement are used here to accurately determine the NGD center frequency $f_{n}$, and the NGD bandwidth $B_{n}$.

- Step 2: The second step of the BP NGD analysis is the definition of the input signal required for the test and denoted $v_{i n}(t)$. Appropriate signals for the TD BP NGD analysis are impulse signals since they make easier the determination of the envelope propagation delay compared to usual modulated carriers. Nevertheless, their spectrum must be centred on $f_{n}$ and their bandwidth must be lower or equal to $B_{n}$. For the case of microwave circuits, the expected configuration of the TD test setup is constituted by a $50 \Omega$ signal generator, the CUT, and a $50 \Omega$ oscilloscope as shown in Fig. 4.

- Step 3: The experimental setup shown in Fig. 4(a) must respect certain expectations. Firstly, the signal generator and the oscilloscope must have a bandwidth which matches with the input test signal. In addition, to observe in the same time the input and output CUT signals, they must have two channels at least, one to directly transmit the input signal from the generator to the oscilloscope (direct path) and another one to transmit the input signal through the CUT (CUT path). Then, the connectors, and the cables interconnecting the CUT and the equipment's must guarantee an accurate synchronization between the two paths.

- Step 4: The analysis of the transient signal should start from the simultaneous visualization of $v_{\text {in }}$ and $v_{\text {out }}$ defined in Fig. 4(a). If the corresponding envelopes are denoted by $\operatorname{Env}\left(v_{\text {in }}\right)$ and $\operatorname{Env}\left(v_{\text {out }}\right)$, the TD attenuation $b$ can be defined as the input and output maximum envelopes ratio as follows:

$$
b=\max \left[\operatorname{Env}\left(v_{\text {out }}\right)\right] / \max \left[\operatorname{Env}\left(v_{\text {in }}\right)\right] .
$$

Several characteristics can also be used to determine the NGD value from TD measurement such as the times difference when rising or falling edges of the input and output envelopes reach $50 \%$ of their final value. However, the NGD value can equally be characterized by the times difference when the input and output envelopes reach their maximum value as described by the following equation:

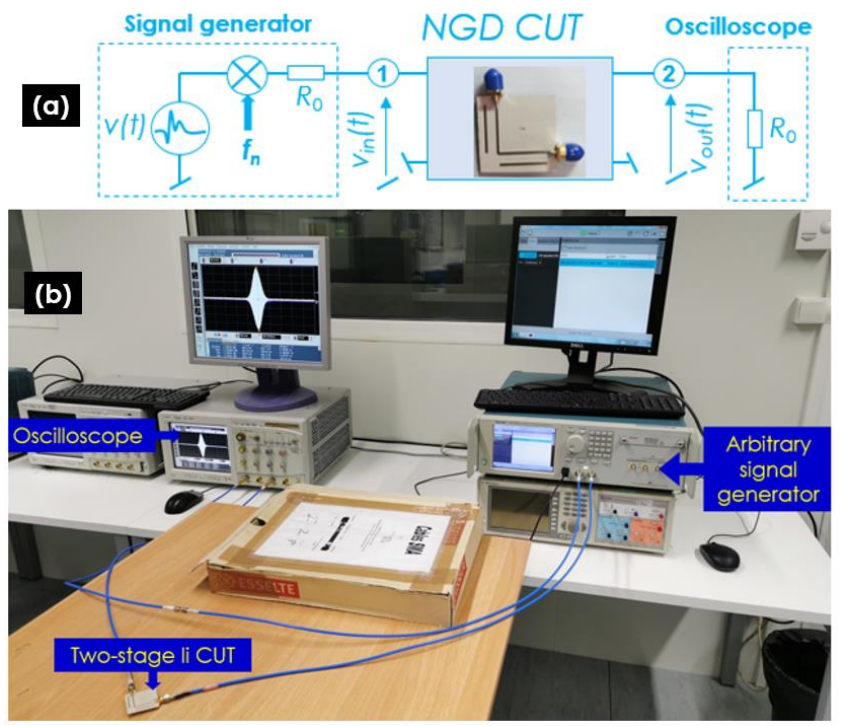

Fig. 4. (a) TD BP NGD test principle and (b) experimental setup used for the double-li CUT.

$$
\Delta t_{\text {peak }}=t_{\text {out }}-t_{\text {in }}
$$

with:

$$
\left\{\begin{array}{l}
\max \left[\operatorname{Env}\left(v_{\text {out }}\right)\right]=v_{\text {out }}\left(t_{\text {out }}\right) \\
\max \left[\operatorname{Env}\left(v_{\text {in }}\right)\right]=v_{\text {in }}\left(t_{\text {in }}\right)
\end{array} .\right.
$$

Fig. 4.b depicts the TD experimental setup used here for the double li CUT. During the test, the ultra-wide band (UWB) microwave arbitrary signal generator Tektronix ${ }^{\circledR}$ AWG70001A, which can generate an arbitrary signal using 10 bits samples with a sampling rate of $50 \mathrm{GSps}$, has been used. This signal generator can deliver two perfectly synchronized opposite signals with a time shift better than 5 ps. To plot the signals, the four-channel oscilloscope referenced Agilent ${ }^{\circledR}$ DSO81204A, which has a bandwidth of $12 \mathrm{GHz}$ and a maximal sampling rate equal to $40 \mathrm{GSps}$, has been used. As stated in the 
previous section, each channel of the signal generator (resp. oscilloscope) has an output (resp. input) impedance of $50 \Omega$.

As indicated previously in step 3, the CUT input port has been connected to one channel of the signal generator and the CUT output port to one channel of the oscilloscope through $1 \mathrm{~m}$ SMA cables (CUT path). The same cables reference has been used to connect one side of a SMA transition to the second channel of the signal generator, and the other side of the SMA transition to another channel of the oscilloscope (direct path). Finally, the TD test has been done by considering a Gaussian up-converted pulse defined in the following subsection as input signal.

\section{B. Input Signal Definition}

In the present study, the chosen input signal is a sine carrier modulated by a Gaussian waveform. This test signal can be analytically defined by:

$$
v_{i n}(t)=\mathrm{e}^{-\xi\left(t-t_{0}\right)^{2}} \sin \left(2 \pi f_{p} t\right) .
$$

where $f_{p}$ is the carrier frequency which can be chosen equal to the NGD center frequency to observe the NGD in TD. From (8), the Gaussian signal reaches its maximal value at the $t_{0}$ time and has a bandwidth $B_{G}$ defined at $\zeta_{d B}$ which can be computed as follows:

$$
\xi=-5 \pi^{2} B_{G}^{2} /\left[\zeta_{d B} \ln (10)\right]
$$

with:

$$
\zeta_{d B}=20 \log (a) .
$$

During the test, it is crucial that the input signal power spectrum is tuned to interleave within the NGD frequency band as illustrated by Fig. 1(b). For this reason, the NGD time-domain experiment must be absolutely preceded by a FD analysis in order to confirm the NGD specifications as aforementioned in section II via the GD response as shown in Fig. 3(a).

Thus, the performed TD measurement tests were carried out using the double-li CUT of Fig. 2(b) for two carrier frequencies and for two Gaussian envelopes having a different bandwidth. The first carrier frequency is $f_{p}=f_{l}=2.345 \mathrm{GHz}$, which corresponds to the minimum GD response frequency of the double-li circuit as shown in Fig. 3(a), and the second one is $f_{p}=f_{P G D}=2.5 \mathrm{GHz}$ where the GD is positive. About envelopes, the first one has a bandwidth of $B_{G 1}=30 \mathrm{MHz}$ with $\zeta_{d B}=10 \mathrm{~dB}$, and the second one has a bandwidth of $B_{G 2}=15 \mathrm{MHz}$ with $\zeta_{d B}=10 \mathrm{~dB}$. The parameters of the test input signals are summarized in Table II.

The following subsection focuses on the transient measurement results obtained with the experimental setup shown in Fig. 4(b).

\section{Analysis of the Time Domain Measurement Results}

During the NGD measurement campaign, the output signal envelope should appear in time-advance compared to the input one. Using the test platform shown in Fig. 4(b), the results shown in Figs. 5(a)-(b) have been obtained for the case of a Gaussian input envelope having a bandwidth $B_{G l}$. Nevertheless, Fig. 5(a) (resp. Fig. 5(b)) corresponds to the case where $f_{p}=f_{1}$ (resp. $f_{p}=f_{P G D}$ ).

\section{1) Transient Measurement Results}

As expected, on the contrary of Fig. 5(b), the output envelope is in time-advance compared to the input one in Fig. 5(a). The double li-circuit TD experimented results must be interpreted as follows:
- In these two screenshot pictures, the yellow curve represents the input signal. Thus, it represents the input Gaussian up-converted pulse as analytically defined in the previous subsection.

- The purple curve represents the output signal and the white curve represents the part where the input and the output signals are superimposed.

- The output signal was rescaled in order to highlight the output signal advance compared to the input.

Thus, at this stage of the experimental study, it has been successfully demonstrated in the TD that the CUT has a NGD around $f_{l}$.

For more didactic analysis of the results, the corresponding csv-format data saved from the Agilent ${ }^{\circledR}$ DSO81204A digital oscilloscope have been post-processed in MATLAB $囚$. Results are shown in Figs. 5(c)-(g). In these graphs, the full signal and its envelope are respectively plotted in red and black as follows:

- the measured input signal specified by the bandwidth-carrier frequency $\left(B_{G 2}, f_{l}\right)$ is shown Fig. 5(c) and the associated measured output signal is displayed in Fig. 5(d),

- the output measured transient signal corresponding to the input signal configuration $\left(B_{G 2}, f_{P G D}\right)$ is shown in Fig. 5(e),

- the input and output measured transient signals under the configuration $\left(B_{G l} f_{l}\right)$ are respectively shown in Fig. 5(f) and Fig. $5(\mathrm{~g})$.

In the next paragraph, the input and output signal envelopes, shown by black curves in Figs. 5(c)-(g) are analyzed to determine the BP NGD specifications from the TD measurement test.

TABLE II

SPECIFICATIONS OF THE TESTED INPUT SIGNAL

\begin{tabular}{c|c|c}
\hline \hline Specifications & Description & Value \\
\hline \hline Amplitude & $V_{\max }$ & $200 \mathrm{mV}$ \\
\hline Bandwidth parameter & $\zeta$ & $-10 \mathrm{~dB}$ \\
\hline \hline \multirow{2}{*}{ Sine carrier frequency } & \multirow{2}{*}{$f_{p}$} & $f_{l}=2.345 \mathrm{GHz}$ \\
\cline { 3 - 3 } & \multirow{2}{*}{ Bandwidth } & $f_{P G D}=2.5 \mathrm{GHz}$ \\
\cline { 3 - 3 } & & $B_{G 1}=30 \mathrm{MHz}$ \\
\hline \hline
\end{tabular}

2) Discussion on the Measured Signal Envelopes

The results from the TD BP NGD study of the two-stage li-CUT are analyzed by comparing input and output envelopes. Figs. 6(a) and 6(b) (resp. Figs. 6(c) and 6(d)) represent the measured and the normalized signal envelopes when a Gaussian envelope having a bandwidth of $B_{G 2}$ (resp. $B_{G I}$ ) is considered. The input and output envelopes corresponding to carrier frequency $f_{l}$ (resp. $f_{P G D}$ ) are respectively displayed using black solid and red (resp. blue) dashed curves.

As predicted by the FD results of Figs. 3 and specified by Table I, it can be emphasized that the outputs corresponding to the carrier frequency $f_{l}$ is significantly attenuated compared to the input. Therefore, the unity-normalized plots of inputs and outputs are shown in Fig. 6(b) (resp. Fig. 6(d)) for the Gaussian with a bandwidth $B_{G 2}$ (resp. $\left.B_{G 1}\right)$. It can be understood from these normalized plots that the rising and falling edges of the output envelopes are in time-advance compared to the input for the carrier frequency $f_{1}$. Nevertheless, for the carrier frequency $f_{P G D}$, the rising and falling edges of the envelope outputs are classically positively delayed compared to the input ones. From these results, it has also been verified that when the input signal 
has a bandwidth in the NGD frequency band, the extraordinary effect of the output envelopes time advance compared to the input ones can be seen. It is equally possible to note that despite the inevitable noise affecting the measured signals, input and output voltages of the double li-circuit remain very well-correlated thanks to a negligible distortion.

The different time-advance signatures for the two considered bandwidths around the dual NGD frequency bands can be understood from the zoom on the input and output envelopes rising edges (resp. falling edges) shown in Fig. 7(e) and Fig. 7(f) (resp. Fig. 7(g) and Fig. 7(h)). The measurement results obtained for every TD test are summarized in Table III.
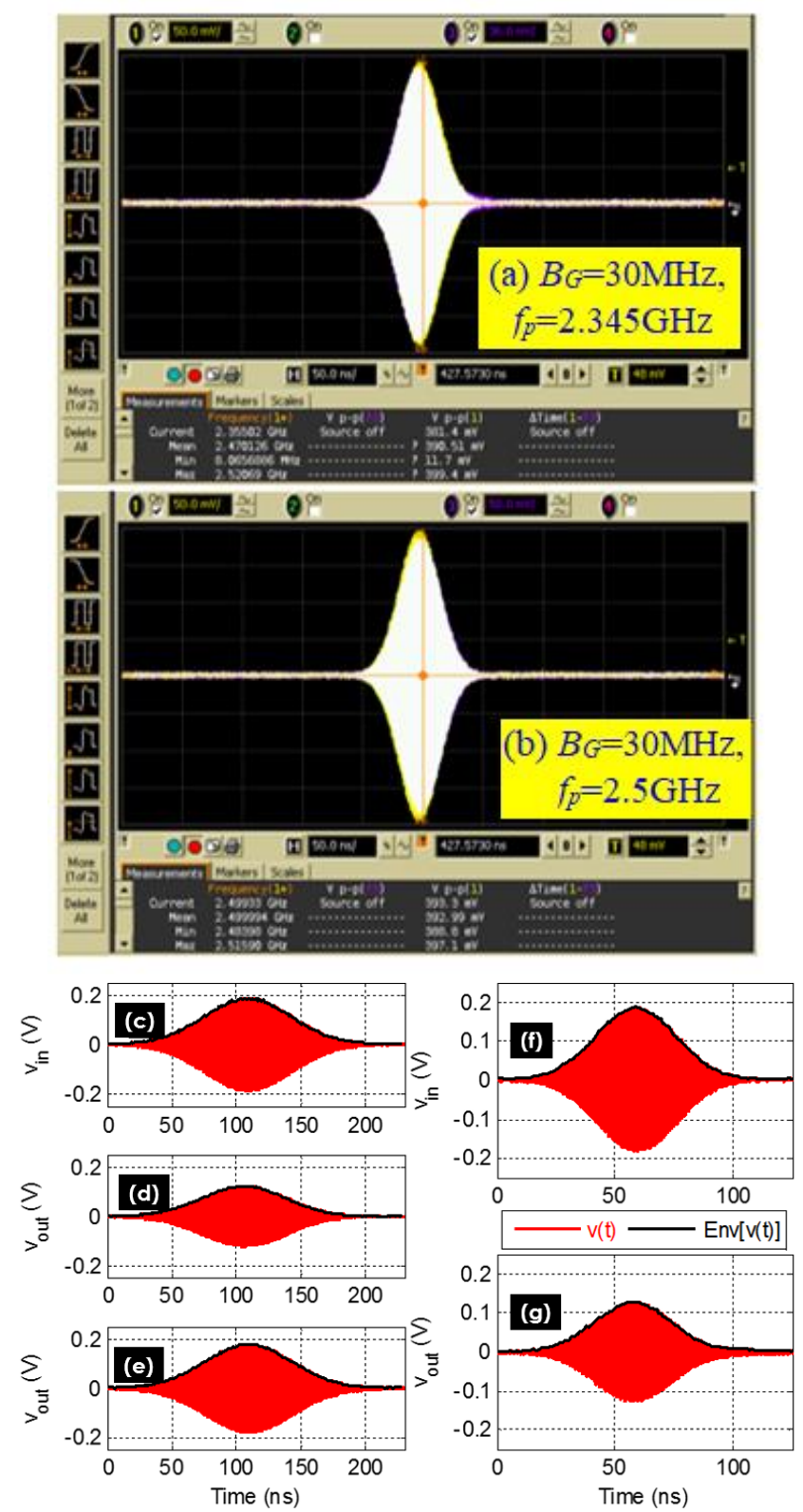

Fig. 5. Screenshots of (a) $\left(B_{G l} f_{l}\right)$ and (b) $\left(B_{G I} f_{P G D}\right)$ input and output Gaussian up-converted pulses. Transient (c) ( $\left.B_{G 2}, f_{l}\right)$-input, (d) (B $\left.B_{G 2}, f_{l}\right)$-output, (e) $\left(B_{G 2}, f_{P G D}\right)$-output, (f) $\left(B_{G I} f_{l}\right)$-input and $(\mathrm{g})\left(B_{G I}, f_{l}\right)$-output signals.

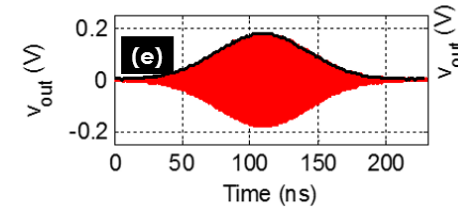

\section{CONCLUSION}

An innovative experimental TD test of BP NGD microwave circuit is investigated. The TD test methodology is explained from the FD S-parameter characteristics of the BP NGD CUT to the input signals and test platform specifications. These specifications, chosen below the BP NGD CUT characteristics, constitute the key point of the presented test.

The developed test is highlighted with a double li-microstrip circuit. The double li-circuit has been analyzed in the FD and shows as expected a dual-band BP NGD. The feasibility of the TD test has been validated experimentally using Gaussian envelopes modulating sine carrier frequencies inside and outside the NGD frequency bands. Thus, it has been verified with the help of experimental results that the double-li circuit output can present rising and falling envelope edges in time-advance compared to input ones when test signal bandwidths are set in the NGD frequency bands.

The proposed study also paves the way to real BP NGD applications and notably for the time-delay compensation by exploiting the GD equalization technique [12].
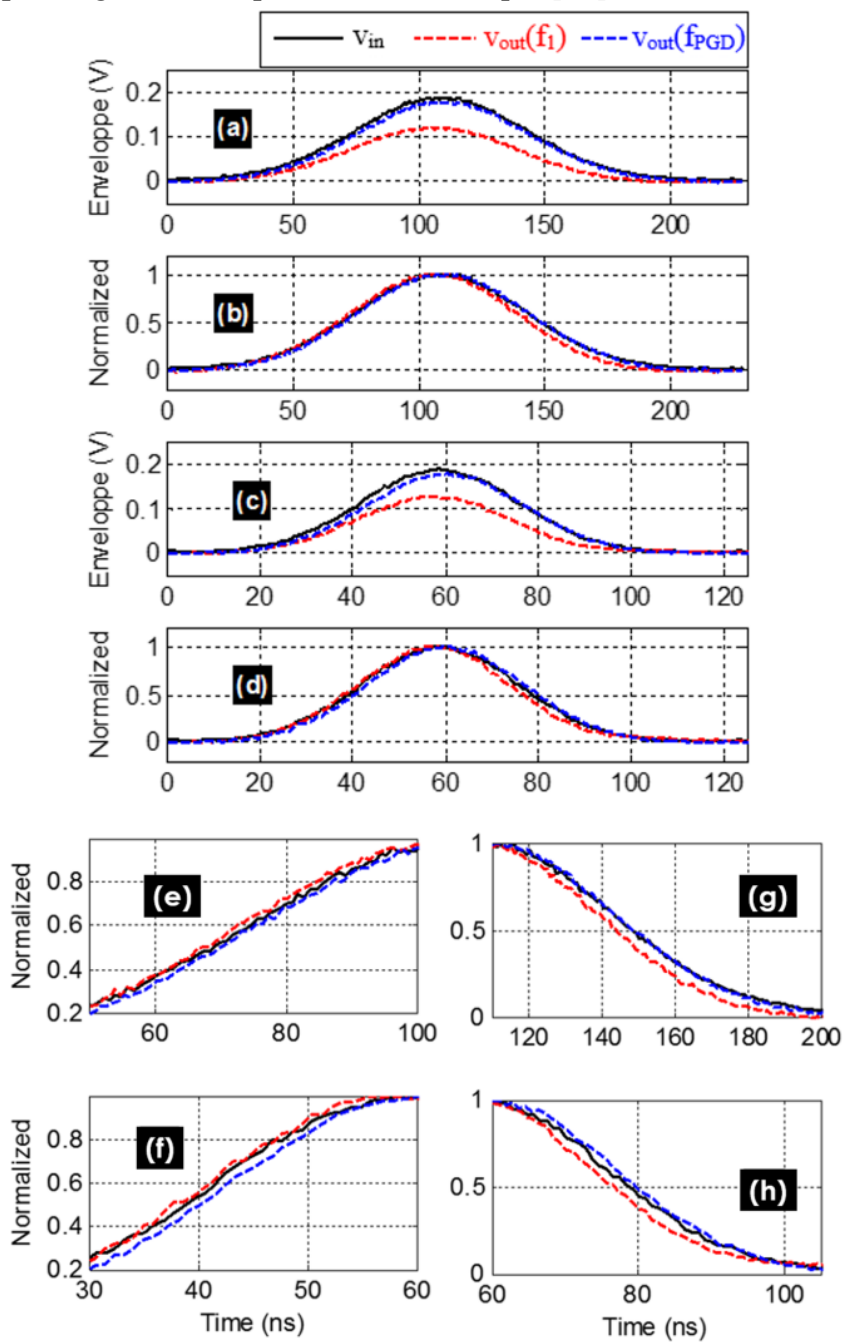

Fig. 6. (a) Measured and (b) normalized Gaussian envelope responses for $\left(B_{G 2}, f_{1}, f_{P G D}\right)$. (c) Measured and (d) normalized Gaussian envelope responses for $\left(B_{G 1}, f_{1}, f_{P G D}\right)$,. Zoom in rising edge normalized envelopes for $\left(B_{G 2}, f_{l}, f_{P G D}\right)(\mathrm{e})$ and $\left(B_{G 1}, f_{l}, f_{P G D}\right)(\mathrm{f})$. Zoom in falling edge normalized envelopes for $\left(B_{G 2}, f_{l}, f_{P G D}\right)(\mathrm{g})$ and $\left(B_{G l}, f_{l}, f_{P G D}\right)(\mathrm{h})$. 
TABLE III

COMPARISON OF THE INPUT AND OUTPUT SIGNAL CHARACTERISTICS FOR THE DOUBLE li-CIRCUIT

\begin{tabular}{|c|c|c|c|}
\hline Specifications & $\begin{array}{c}\text { (Bandwidth, } \\
\text { carrier } \\
\text { frequency) }\end{array}$ & Description & Value \\
\hline Rise time advance & \multirow{4}{*}{$\left(B_{G 2} f_{l}\right)$} & $\overline{\Delta \Delta t_{\text {rise }}}$ & $-2.1 \mathrm{~ns}$ \\
\hline Fall time advance & & $\Delta t_{\text {fall }}$ & $-3.9 \mathrm{~ns}$ \\
\hline Voltage attenuation & & $b$ & 0.64 \\
\hline Correlation coefficient & & $\operatorname{corr}\left(V_{\text {in }}, V_{\text {out }}\right)$ & $99.5 \%$ \\
\hline Rise time advance & \multirow{4}{*}{$\left(B_{G I}, f_{1}\right)$} & 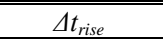 & $-0.9 \mathrm{~ns}$ \\
\hline Fall time advance & & $\Delta t_{\text {fall }}$ & $-1.8 \mathrm{~ns}$ \\
\hline Voltage attenuation & & $b$ & 0.68 \\
\hline Correlation coefficient & & $\operatorname{corr}\left(V_{\text {in }}, V_{\text {out }}\right)$ & $96.2 \%$ \\
\hline Rise time advance & \multirow{4}{*}{$\left(B_{\mathrm{G} 2}, f_{P G D}\right)$} & $\Delta t_{\text {rise }}$ & $1.7 \mathrm{~ns}$ \\
\hline Fall time advance & & $\Delta t_{\text {fall }}$ & $0.6 \mathrm{~ns}$ \\
\hline Voltage attenuation & & $b$ & 0.96 \\
\hline Correlation coefficient & & $\frac{D}{\operatorname{corr}\left(V_{\text {in }}, V_{\text {out }}\right)}$ & $99.9 \%$ \\
\hline Rise time advance & \multirow{4}{*}{$\left(B_{G l}, f_{P G D}\right)$} & $\Delta t_{\text {rise }}$ & $1.8 \mathrm{~ns}$ \\
\hline Fall time advance & & $\Delta t_{\text {fall }}$ & $0.8 \mathrm{~ns}$ \\
\hline Voltage attenuation & & $b$ & 0.97 \\
\hline Correlation coefficient & & $\operatorname{corr}\left(V_{\text {in }}, V_{\text {out }}\right)$ & $99.8 \%$ \\
\hline
\end{tabular}

\section{REFERENCES}

[1] A. Kumar and Y. Bar-Shalom, "Time-domain analysis of cross correlation for time delay estimation with an autocorrelated signal," IEEE Transactions on Signal Processing, vol. 41, no. 4, pp. 1664-1668, April 1993.

[2] S. Kouhpayeh-Zadeh-Esfahani, A. Abdipour, and K. Afrooz, "Fast Methodology for Time-Domain Analysis of Nonlinear-Loaded Transmission Line Excited by an Arbitrary Modulated Signal," Computer-Aided Design of Integrated Circuits and Systems IEEE Transactions on, vol. 39, no. 3, pp. 670-674, 2020.

[3] A. Sánchez-Alegría, P. Moreno, J. R. Loo-Yau, and S. Ortega-Cisneros, "Time Domain Electromagnetic Transient Analysis of Aerial Nonuniform Transmission Lines Excited by an Incident Electromagnetic Field," Mathematical Problems in Engineering, vol. 2019, pp. 1, 2019.

[4] S. Ben-Yaakov, S. Glozman and P. Rabinovici, "Envelope simulation by SPICE-compatible models of linear electric circuits driven by modulated signals," IEEE Trans. Ind. Electron., vol. 37, no. 2, pp. 527-533, Mar./Apr. 2001.

[5] Y. Chang, "Transient analysis of lossless-coupled transmission lines in a nonhomogeneous dielectric medium," IEEE Trans. Microwave Theory Tech., vol. MTT-18, pp. 616-626, Sept. 1987.

[6] D. Y. Heh, and E. L. Tan, "Multiple LOD-FDTD Method for Multiconductor Coupled Transmission Lines," Multiscale and Multiphysics Computational Techniques IEEE Journal on, vol. 5, pp. 201-208, 2020.

[7] B. Ravelo, L. Wu, F. Wan, W. Rahajandraibe and N. M. Murad, "Negative Group Delay Theory on li Topology," IEEE Access, Vol. 8, No. 1, Mar. 2020, pp. 47596-47606.

[8] F. Wan, B. Liu, P. Thakur, A. Thakur, S. Lalléchère, W. Rahajandraibe, and B. Ravelo, "OIO-Shape PCB Trace Negative Group-Delay Analysis," IEEE Access, Vol. 8, No. 1, Dec. 2020, pp. 2169-3536.

[9] X. Zhou, B. Li, N. Li, B. Ravelo, X. Hu, Q. Ji, F. Wan and G. Fontgalland, "Analytical Design of Dual-Band Negative Group Delay Circuit with Multi-Coupled Lines," IEEE Access, Vol. 8, No. 1, Apr. 2020, pp. 72749-72756.

[10] B. Ravelo, "Similitude between the NGD function and filter gain behaviours," Int. J. Circ. Theor. Appl., vol. 42, no. 10, Oct. 2014, pp. 1016-1032.

[11] F. Wan, J. Wang, B. Ravelo, J. Ge, and B. Li, "Time-Domain Experimentation of NGD Active RC-Network Cell," IEEE Transactions on Circuits and Systems II: Express Briefs, Vol. 66, No. 4, Apr. 2019, pp. 562-566.

[12] B. Ravelo, S. Lalléchère, A. Thakur, A. Saini and P. Thakur, "Theory and circuit modelling of baseband and modulated signal delay compensations with low- and band-pass NGD effects," Int. J. Electron. Commun. (AEÜ), Ed. Elsevier, Vol. 70, No. 9, Sept. 2016, pp. 1122-1127.
R. Vauche received the Ph.D. degree in microelectronics from Aix-Marseille University, France, in 2011. Since 2014, he has been an Associate Professor at IM2NP, Aix-Marseille University, France. His research interests include the design of integrated circuits and systems for ultra-wideband impulse radio, human body communications, and home-care applications.

R. Assila Belhadj Mefteh received the M.S. degree in embedded electronics from the University of Lorraine, Nancy, France, in 2019. Since 2019, he has been a microelectronics Ph.D. student at IM2NP, Aix-Marseille University, France. His research interests cover the design of emitters and receivers for human body communications.

F. Haddad received the $\mathrm{Ph}$.D. degree in microelectronics from Aix-Marseille University, France, in 2009. Since 2010, he has been an Associate Professor at IM2NP, Aix-Marseille University, France. Her research interests include CMOS analog and RF integrated circuits design, ultra-low power, and multi-standards applications. She is IEEE member.

W. Rahajandraibe received the Ph.D. degree in microelectronics from University of Montpellier, France, in 2002. Since 2014, he has been a Professor at IM2NP, Aix-Marseille University, France. His current research interests include the design of ultralow power circuit and system for smart sensor interface, e-health, wireless systems, and multi-standard transceiver.

Fayu WAN received the Ph.D. degree in electronic from the University of Rouen, Rouen, in 2011. He is currently a Full Professor with the Nanjing University of Information Science and Technology, Nanjing, China. His current research interests include negative group delay circuits, electrostatic discharge, electromagnetic compatibility, and advanced RF measurement.

S. Lalléchère received the $\mathrm{PhD}$. Degree in electromagnetics from Blaise Pascal University, Clermont-Ferrand, France, in 2006. Since 2007, he has been an Associate Professor at Institut Pascal and Universite Clermont Auvergne, France. His research interests cover the fields of electromagnetic compatibility including complex electromagnetic environments, and computational electromagnetics.

G. Fontgalland (S'97-A'99-M'00-SM'07) is a Full professor at Federal University of Campina Grande, Brazil. His research interest is on: EM modeling, EMC, EMI, RFID, propagation, and antennas for various applications. He has published more than 200 papers in journal and conferences. He is senior IEEE member.

Prof. Preeti Thakur, Ph.D. in Physics and a Gold medalist in Masters in Physics (Electronic Science), is currently Head, Department of Physics at Amity University Haryana, Gurgaon, India. Her research interests are negative group delay circuit, nanomaterials synthesis and characterization for high frequency applications, radar absorbing materials and sensor application. 
Prof. Atul Thakur is M.Phil and Ph.D. from Himachal Pradesh University, Shimla, India. He has successfully completed several government funded projects. He is working as Director, Amity Institute of Nanotechnology, Amity University Haryana, India. His current research area is negative group delay and filed eighteen patents till date.
Prof. Blaise Ravelo (M'09) is currently Full Professor at NUIST, Nanjing, China. His research interest is on multiphysics and electronics engineering. He is a pioneer of the negative group delay concept. He is member of IET Electronics Letters editorial board and member of IEEE, URSI, GDR Ondes, and Radio Society. 